"Sex Will Make Your Fingers Grow Thin and Then You Die": The Interplay of

Culture, Myths, and Taboos on African Immigrant Mothers' Perceptions of Reproductive Health Education with Their Daughters Aged 10-14 Years Kafuli Agbemenu, Margaret Hannan, Julius Kitutu, Martha Ann Terry \& Willa Doswell

Journal of Immigrant and Minority Health

ISSN 1557-1912

$\mathrm{J}$ Immigrant Minority Health DOI 10.1007/s10903-017-0675-4

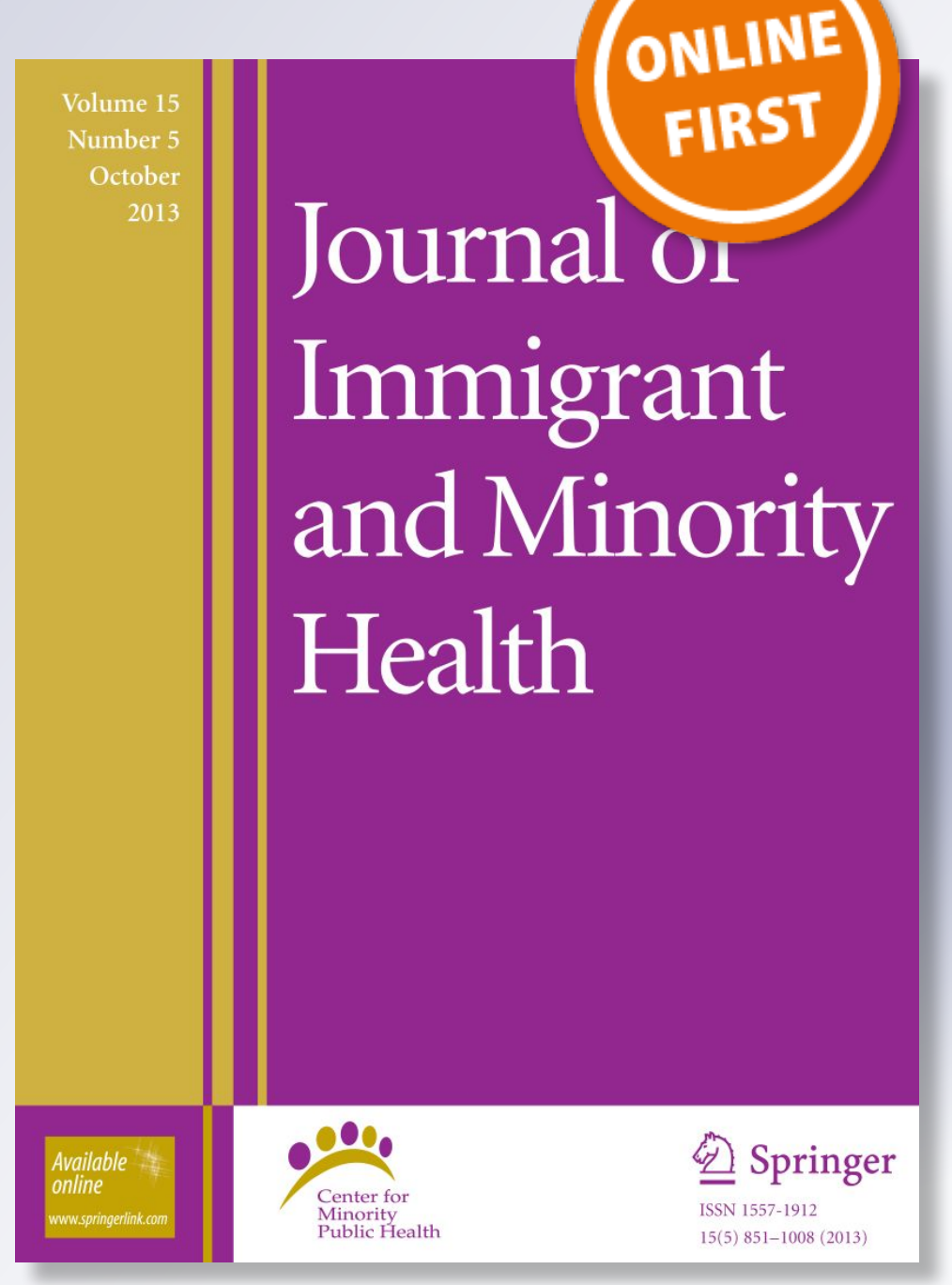

望 Springer 
Your article is protected by copyright and all rights are held exclusively by Springer Science+Business Media, LLC, part of Springer Nature. This e-offprint is for personal use only and shall not be self-archived in electronic repositories. If you wish to selfarchive your article, please use the accepted manuscript version for posting on your own website. You may further deposit the accepted manuscript version in any repository, provided it is only made publicly available 12 months after official publication or later and provided acknowledgement is given to the original source of publication and a link is inserted to the published article on Springer's website. The link must be accompanied by the following text: "The final publication is available at link.springer.com". 


\title{
"Sex Will Make Your Fingers Grow Thin and Then You Die": The Interplay of Culture, Myths, and Taboos on African Immigrant Mothers' Perceptions of Reproductive Health Education with Their Daughters Aged 10-14 Years
}

\author{
Kafuli Agbemenu' ${ }^{1} \cdot$ Margaret Hannan $^{2} \cdot$ Julius Kitutu $^{3} \cdot$ Martha Ann Terry $^{4} \cdot$ Willa Doswell $^{5}$
}

๑) Springer Science+Business Media, LLC, part of Springer Nature 2017

\begin{abstract}
This paper examines the convergence of culture, myths, and taboos surrounding reproductive health issues African immigrant women, living in the United States, learned during childhood in their countries of origin. We also discuss how mothers' perceptions of reproductive health education (RHE) influenced the education of their own daughters aged 10-14 years. This was a qualitative descriptive study. Data were collected via interviews and demographic survey. The sample size was 20 African immigrant mothers living in a mid-sized city in the U.S. Interviews were transcribed verbatim. Qualitative data was analyzed using qualitative content analysis. Myths and taboos related to menstruation, sexual intercourse, pregnancy, and HIV/AIDS were reported by the women interviewed. Discussion of these issues was largely taboo, and most myths the mothers learned growing up pertained to sexual intercourse, pregnancy prevention, and pregnancy termination using nonhormonal ingested substances. Myths and taboos about sexual issues are widespread in Africa and are propagated to control sexual behavior, especially that of unmarried people, particularly women. By examining these myths and taboos, we are able to somewhat contextualize the mothers' immigrant experience regarding RHE. Although myths were reported, the majority of mothers did not appear to believe them. The most significant taboo reported was sexual intercourse. This in turn led to mothers' overemphasis on abstinence for their daughters. It is also noteworthy that this sample contained mainly African women who overall were highly educated, spoke English, and could adequately navigate life in the U.S. It is unclear what the results would be if we were to examine African immigrant women with less achievements in these areas.
\end{abstract}

Keywords African immigrants · Reproductive health education · Adolescents · Mothers · Qualitative research

Kafuli Agbemenu

agbemenu@buffalo.edu

Margaret Hannan

meghannan50@gmail.com

Julius Kitutu

jmm@ @itt.edu

Martha Ann Terry

materry@pitt.edu

Willa Doswell

wdo100@pitt.edu
2 School of Nursing, University of Pittsburgh, Pittsburgh, USA

3 Department of Acute and Tertiary Care, School of Nursing, University of Pittsburgh, 239 Victoria Building, 3500 Victoria Street, Pittsburgh, PA 15261, USA

4 Behavioral and Community Health Sciences, School of Public Health, University of Pittsburgh, 222 Parran Hall, 130 De Soto Street, Pittsburgh, PA 15261, USA

5 Department of Health Promotion \& Development, School of Nursing, University of Pittsburgh, 440 Victoria Building, 3500 Victoria Street, Pittsburgh, PA 15261, USA

1 Department of Family, Community, \& Health Systems Sciences, School of Nursing, , State University of New York, University at Buffalo, 3435 Main Street, Room 201D, Buffalo, NY 14214, USA 


\section{Background}

According to the 2010 U.S. census, there are approximately 1.7 million documented African immigrants in the United States, who make up $4 \%$ of the foreign born population [1]. Overall, Africans immigrants in the U.S. typically adjust to the culture through the process of acculturation. Acculturation is when individuals who have grown up in one cultural environment adapt to new environments that result from migration and exposure to a majority population [2]. However, undergoing the process of acculturation neither negates the culture of origin nor its attendant and continued beliefs in myths and taboos concerning various issues [3].

In historical accounts from some African societies predating Christianity, it is reported that children were socialized to their adult responsibilities as they approached puberty [4]. This socialization included sex education. Because it was considered taboo for sex to be discussed between immediate family members, this education was led by grandparents. Grandparents taught their grandchildren about gender-based marital expectations and sexual behavior. For example, young people in Kenyan and Ghanaian traditional societies took part in initiation rites, after which they were considered adults and were free to marry. In Ghana, the ceremony known as Dipo is an initiation rite of the Krobo people. Dipo is conducted after a girl's first menstruation. During Dipo, these girls participated in ritual tasks such as bathing and a week-long seclusion period. They were also taught about sexual intercourse and birth control [5]. In other initiation rites, initiates received more in-depth sex education through the use of sexually explicit language and dances [4]. These actions were guided by a strict moral code.

With the introduction of Christianity, many traditional coming-of-age rituals were eliminated. An example is Kenya, where missionaries arrived in the 1800s. These missionaries were extremely conservative in dress, language, and dance and their behavior conflicted with the indigenous practices they encountered. African cultural practices were denounced and began to decline [4]. Christian/European practices were then increasingly incorporated into local culture, resulting in a casade of negative event-adolescent girls were less likely to be involved in initiation rites, and their parents, extended family, teachers, and other adult role models became uncomfortable teaching girls responsible sexual behavior $[4,6]$.

Myths and, to a lesser extent, taboos about sexual issues are widespread in African countries. They are propagated in an effort to control sexual behavior, especially the sexual behavior of unmarried people and-even more so-women [7-9]. In this study, myth is defined as a widely held but false belief in something-in this case, sexual issues. This definition is congruent with Amanze's description of the creation myths of African people in which the development of human sexuality emerges [10]. In Amanze's discussion of myths, he highlights the fact that conversations of a sexual nature typically used euphemisms and were usually shrouded in secrecy. In this study, taboo is defined as reported sexual behavior prohibited or restricted by social custom. This study further describes reproductive health education (RHE) as talking about topics related to having children such as reproductive body parts, monthly periods, how pregnancy happens, and how to prevent pregnancy.

To begin to understand the phenomenon of motherdaughter reproductive health education in the African immigrant population living in the U.S., a review of studies from African countries including Kenya, Ghana, and Nigeria was conducted. This review was conducted because no U.S. based studies examining the study phenomenon in African immigrants were identified. In the studies reviewed, reproductive health education in some form took place in the early preteen years, starting at about age 10. Parental factors that influenced communication included: education level, lack of knowledge about menstruation and pregnancy prevention, religiosity, previous reproductive health communication with their own parent, and cultural norms [4, 8-11]. Ironically, in these studies, higher levels of education did not correlate with increased communicative ability $[4,9]$.

Reproductive health was discussed with great reluctance in unidirectional (one-way, parent-to-child) conversations. Fear tactics were used to ensure that teenage girls remained abstinent until marriage. These findings are congruent with reports on parent-child communication on sexual issues in some African countries [3, 7, 8]. In the Biddlecom et al. study conducted in Burkina Faso, Ghana, Malawi, and Uganda, parents or parental figures were seldom involved in conversation about sex-related matters or information on contraceptives [12].

Izugbara explored parent-child communication in rural Nigeria where parents instilled fear about sex to scare children from sexual activity. Condoms, contraceptives, and abortions were rarely mentioned. Parents seldom encouraged use of condoms and contraceptives, because they felt that mentioning condoms and contraceptives would lead to waywardness and promiscuity [11].

Mbugua examined RHE provided by educated urbandwelling mothers in Kenya. She discovered that though mothers had high levels of formal education, they did not have fact-based conversations about sex with their daughters. Mothers resorted to scare tactics to shut down any reproductive health communication [13].

Overall, parents were reluctant to provide sexual health information because they believed it caused children to 
begin engaging in sexual behavior or promiscuity [9-11]. When reproductive education was provided, it was a unidirectional process along gender lines. Mothers talked to daughters, and fathers talked to sons. Parents gave instructions about expected sexual behavior and did not encourage discussion or questions. Reproductive health education was given instead in the form of warnings, threats and passing on of sexual myths and taboos aimed at instilling fear $[9,10,13-16]$. In order to understand factors that influence how African immigrant mothers living in the United States provide reproductive health education (RHE) to their teenage daughters, an examination of the sexual myths and taboos the mothers grew up with was conducted.

\section{Methods}

This study used a qualitative descriptive approach. This methodology allows for interpretation of the phenomenon without modification. It is useful for analyzing phenomena for which we have little research, or for studying marginalized populations [17]. The research was conducted in accord with prevailing ethical principles and reviewed by an Institutional Review Board.

\section{Data Collection}

This study was inspired by the lived experience of the study $\mathrm{PI}$-an African immigrant, who grew up exposed to reproductive health myths and taboos herself that led to negative health outcomes for those involved.
A purposive sample of participants were recruited from the community, mostly via word of mouth and in collaboration with African businesses, organizations and community meetings. Businesses and organizations included African grocery stores, hair salons, churches, and a local organization that advocates for African immigrants. The PI attended numerous community country meetings to recruit participants. Flyers were initially developed for recruitment, but were found to be ineffective in recruitment because potential participants preferred to hear about the study in face to face meetings. Inclusion criteria included women who self-identified as African immigrants, had a daughter 10-14 years, and had conducted reproductive health education discussions with the daughter. Exclusion criteria included African immigrant women with a daughter ages 10-14 years, who had a developmental disability. The age range (10-14 years) was set to coincide with the beginning of menarche and sexual debut, which, as recommended by the literature, is the most ideal time to begin reproductive health education $[11,18]$.

Data were collected via a demographic questionnaire and an in-depth semi-structured interview that were completed in one session [16]. Demographic questionnaires prepared at a sixth grade reading level were administered in paper form. All study documents were in English. No translation was needed as it was study inclusion criteria to be able to read, write, and speak English. An interview guide was developed from literature on parent-child sexual health communication in select African countries (see Table 1) [4, 8-13]. The select African countries from which literature was reviewed included: Ghana, Kenya and Nigeria. These are the top three African countries that contribute immigrants to the U.S [1].
Table 1 Interview questions and probes
Interview questions with probes

1. Did you receive reproductive health education from your mother?
-If yes, tell me what she told you
-Tell me what topics did you talk about?
-If no, where did you learn about reproductive health issues?
2. In general, tell me about how boy girl sexual issues were talked about in (country of origin)
3. Did you hear of any myths or taboos about sexual matters as you were growing up?
-(If yes), tell me more about that
4. Did girls you knew growing up get pregnant?
-If yes, what happened?
-How did you find out?
-How was it dealt with?
-Did you tell your mother about it?
5. Did girls you knew growing up get a sexual disease?
-If yes, what happened?
-How did you find out?
-How was it dealt with?
-Did you tell your mother about it?
6. What do you think about mothers providing this kind of education to their daughters? 
Verbal consent was obtained prior to study interaction. Each participant was interviewed once, for 1-1.5 hours. Only mothers were recruited because they are traditionally gatekeepers of reproductive health education, and because RHE tends to occur along gender lines. All participants were recruited, gave consent, and were interviewed by the PI.The PI is an African immigrant, as such the PI was familiar with aspects of cultural competency based on lived experience. To prepare to conduct interviews, the PI completed doctoral level qualitative methods coursework which included instruction of conducting focus groups and interviews. Participants were approached by the PI directly when participating in community activities that had been facilitated by community leaders, and also via snowball technique.

Interviews took place at a location determined by the participant. Most interviews were conducted in participants' homes, two in the PI's car, and one over the phone. To ensure confidentiality, all participants were asked to ensure there was privacy, and minimal interruptions for the interview. Only the PI and participant were present during interviews. To ensure participants had the same notion of RHE as the researchers, participants were provided with the study definition of RHE prior to initiation of the interview.

\section{Data Analysis}

Qualitative content analysis was used to analyze interview data. This approach employs the study of a phenomenon without altering the environment of the participant or placing limitations on the outcome of the research [19]. It is an ideal technique for the comprehensive examination of health issues in a cultural context, especially in racial and ethnic groups that are not typically visible in research [19-21]. Conducting a qualitative descriptive study is also timely and appropriate because of the paucity of information about African immigrants in the United States, especially concerning reproductive health myths and taboos.

For the qualitative data analysis, transcripts were reviewed one at a time and coded independently by the study $\mathrm{PI}$ and a $\mathrm{PhD}$ trained in qualitative study methods. A code book was developed after review of the first interview, and was used in coding subsequent interviews. After independent coding of each interview, the PI and second coder met to review all coding. All discrepancies were discussed until agreement. Participants selected a pseudonym that has been used when direct quotes are mentioned.

\section{Results}

Twenty immigrant African mothers from seven countries participated. These countries (and the corresponding number of participants) were: Nigeria $(n=10)$, Kenya $(n=4)$,
Guinea $(\mathrm{n}=2)$, Ghana $(\mathrm{n}=1)$, Zambia $(\mathrm{n}=1)$, Liberia $(n=1)$, and South Sudan $(n=1)$. Participants from Nigeria were recruited from a women's group at an African church. Age range was 30-55 years. Average length of stay in U.S. was 13.7 years (range $=2-28$ years). Eighty percent were married and $95 \%$ of participants had some college education or higher. Seventy-five percent $(n=16)$ were Christians, $10 \%$ Muslim ( $\mathrm{n}=2)$, and $10 \%$ chose not to answer this question. Nineteen participants came to the United States on the diversity lottery and for family reunification. One participant came as a refugee.

Overall, this was a well-educated sample of African women who primarily immigrated to the U.S. for better financial and educational opportunities. Three topics were discussed: (1) taboos, (2) myths, and (3) perceived U.S. sexual norms reported by Mothers.

\section{Taboos}

There was limited discussion on taboos. Mothers reported that children were not expected to have any questions about sex. We also started to see the use of scare tactics- - "you have sex with a boy you're going to get pregnant." Pregnancy will not occur with every incident of sexual intercourse, but this statement was used by parents of the mothers to instill fear and to halt all conversation about sex. These views were echoed by study participants, as recounted by one mother:

my parents wouldn't talk to me about sex. It was just a taboo... you have sex with a boy you're going to get pregnant; it's a disgrace to the family; it's a no no; you don't talk about it; you don't question it. Just don't do it... (Auntie, Ghana, June 13)

There was more robust discussion about myths. Four categories of myths were identified: those related to menstruation, sexual intercourse, HIV/AIDS and pregnancy. In this section we will provide examples of the different categories, and attempt to explain the societal function or purpose of the myth.

\section{Myths}

\section{Myths Related to Menstruation}

Menstruation myths revolved around witchcraft and superstition. It was believed that, if sanitary towels were not properly disposed of, they could be used in ritual practices to cast spells or curses on others. Although, it can also be thought that these myths exist to ensure proper hygiene by scaring the menstruating female into proper dispose of used sanitary pads: 
if you leave your sanitary pad then someone can use it to make voodoo. (Barbara, Nigeria, April 12)

\section{Myths Related to Sexual Intercourse}

Some myths involving supernatural happenings, in which humans are believed to adopt animal behaviors or animal forms, use fear to deter people from socially unacceptable behavior. Sexual intercourse myths involved negative consequences of premarital or extramarital affairs, including extreme body changes, supernatural happenings, or even death:

I've heard about people saying that if somebody go and sleep with somebody's wife they are going to crow. They will start cro-co-co-co ... Uh, (like a) hen. Like three times they will do cro-co-do, and then you pass away. (Kehinde, Nigeria, June 01).

I was told, you are not allowed to sleep with a boy; you can't have sex with a boy before you get married. If you decided to do that, you are going to get sick, you are going to get skinny and your fingers they are going to be so long ... I mean your fingers, would get skinny. You'll start looking so bad ... like you were going to look like someone old ... then you get too long and you end up dying... (Kasande, Zambia, April, 03)

\section{Myths Related to HIV/AIDS}

Further conversation with Kasande revealed that the above quote was probably a description of late-stage HIV/AIDS, marked by weight loss and cachexia (muscle wasting), giving the appearance of looking "like someone old ... and you end up dying" (April, 03).

\section{Myths Related to Pregnancy}

Pregnancy myths involved misinformation about how pregnancy occurs, pregnancy prevention, and termination of an unwanted pregnancy. Only one mother reported being aware of modern means of contraceptives while she was growing up. She explained that:

When we were doing biology ...they had written about safe days. Some girls took it literally, and they went ahead and had sex. They get pregnant, and they said they used the biology book not knowing that it's a myth. Safe days are not really like that; there is so much involved with safe days. (Tulip, Kenya, April, 05)

One of the examples of inaccurate RHE was offered by Tulip, who mentions that girls had a literal understanding of safe days, without grasping that cycle lengths varied for each person. In the description above, girls between the ages of 14 and 16 years failed to understand how to calculate their menstrual cycle, which resulted in unwanted pregnancies. As expressed by one of the mothers, there was also misinformation on how to prevent pregnancy:

This [misinformation] were created by men who tried to cheat the girls; they did tell them if you take some kind of medicine it would prevent pregnancy, and some girls would take it, not knowing they were not contraceptive pill. (Tulip, Kenya, April, 05)

A participant perceived uneducated girls as becoming pregnant after taking pills falsely passed off by men as contraceptives. However, considering variable levels of education on pregnancy prevention, it is unclear if the men also believed that the pills they were giving the girls would be effective in preventing pregnancy. There was also the belief that pregnancy could be prevented by drinking undiluted hard liquor. One mother told us:

If you have sex, you want to take alcohol, that you don't want pregnancy to stay, do some Bacardi, yeah, the strong ones. (Shola, Nigeria, May, 21)

Pregnancy termination myths were prolific due to lack of accurate RHE and access to modern methods of birth control. In most myths, pregnancy could be terminated by drinking corrosive or high acidic content substances. One mother explained:

I just knew what they said: if you get pregnant, you grind a bottle... You grind it very smooth, it will cause the baby to come out. (Auntie, Ghana, June, 13)

Myths involving similar abortifacients were reported from different countries, such as in the myth below from Nigeria and Guinea in which lemon was used to terminate pregnancy. A mother from Nigeria reported:

lemon and lime, because they're very strong if you drink it; if you think you're pregnant, you'll flush it down... and "cone" ... it's used to cook ... it's a green leaf...you just put it a little drop ... (Shola, Nigeria, May, 21)

A similar process was described by another mother from Guinea:

drinking Coca-Cola and putting a lot of lemon, to flash the pregnancy, drinking a lot of carbonated drinks. (Nan, Guinea, June, 18)

Another mother told a similar story:

Limestone, they use it to cook, it can burn you in the tongue if you touch it ...then blue, when you wash white clothes and you put blue to make it look whiter, 
that blue, they mix it and drink ... can destroy the pregnancy and cause an abortion. (Janet, Nigeria, July, 16)

Participants also reported benign myths related to sex and pregnancy. These myths may have been propagated as a form of birth control or for child spacing. For example:

I also heard-there are certain times of the season you don't have sex, even though you are husband and wife. You have to be separate. (Kehinde, Nigeria, June, 12).

In this myth, the participant reported that married couples were barred from having sex during the farming season. Sexual abstinence might have been supported to encourage people to focus their energies on farming, and maybe by extension, to ensure that children were born in the harvest months, when food was in plentiful supply.

The myth below cautions pregnant women against excessive sun exposure. Nigeria has a hot weather. The researchers think that this myth was circulated to prevent prolonged sun exposure, which can cause dehydration or heat stroke, negatively affecting the pregnancy:

Between two (p.m.) and four (p.m.) you are not allowed to walk in the streets while the sun is so high, they believe the evil spirits are gonna get into the pregnant woman and then you are gonna give birth to something, maybe a monster or a bad child (Janet, Nigeria, July, 16)

\section{Perceived U.S. Sexual Norms Reported by Mothers}

Mothers felt that U.S. society was more permissive and accepting of overt sexual behavior than their countries of origin. Sexual behavior was believed to be engaged in early, casually and without remorse. Mothers may have perceived U.S. sexual norms as permissive due to their religious beliefs and cultural upbringing in Africa. For example, one mother said:

Sex is not a big deal to Americans. It's like sex is a way of life so for someone who is not your boyfriend, you can have sex with them, but that is not acceptable in Africa, even with your boyfriend, you feel bad because you are not married, that's what leads to separation between boyfriend and girlfriend, but here it's the number one thing on the table. (Barbara, Nigeria, April, 12)

To us, that's not something (sex) that you are supposed to do until you get married. But when I first came to this country, I have a neighbor, to her it was so exciting for her daughter to have a boyfriend at the age of 8 years old. I found it so weird, but to them...it's nothing; (Kitty, Nigeria, June, 04)
As a result of the perceived permissiveness of sexual behavior in American society, mothers mentioned feeling more protective of daughters, and being open to providing reproductive health education to their daughters at earlier ages than they would have if still in their country of origin, and in some instances initiating conversation or seeking out other sources of information for their daughters.

I think especially for us Africans we still have that bad mentality of sex, we still need to make it open to our children. They are trying to do things on their own without us because we still have not accepted it that we are in a different world and different times not like when we were growing up. We need to lay it open and let them know that this is how it is. Thinking that they won't have sex because you tell them is bad; they still have it... We don't want to tell them and think that we tell them bit by bit they leave it (Tulip, Kenya, April 05).

\section{Discussion}

This study was inspired by the lived experience of the PI growing up in an African country where the PI was exposed to several cases of teenage pregnancy resulting from inaccurate reproductive health education. Some cases ended in abortions and suicides. As a healthcare provider, the PI rationalized that it was important and urgent to examine reproductive health education African mothers provided their daughters.

This study was also inspired by a myth the PI heard a few years ago from a Cameroonian woman in the African immigrant community. The woman believed that drinking a glass of her urine every morning would protect her from HIV/ AIDS. We were concerned about other reproductive health myths and taboos that were adhered to, and that could lead to negative health outcomes. Hence we decided to undertake this study. Because the woman mentioned HIV/AIDS, which is primarily spread through sexual intercourse, this is a reproductive health concern. The PI decided to investigate information that African immigrant women were exposed to, and what information they passed on to their daughters entering puberty and potential sexual debut.

Mothers in our study were from cultures that did not condone premarital sex - or even boy-girl relationships-in adolescence [17]. Although it might appear contradictory, as per conversation with the mothers, an element of protectiveness was to be open with their daughters. The mothers articulated a desire to ward off negative consequences of sexual activity, by providing RHE early/when daughters were at a younger age, and not shutting down conversation initiated by daughters. 
They tended to retain their cultural attitude towards RHE, but they did not maintain cultural actions. This study defined cultural attitudes as mothers' views on reproductive health issues, such as abstinence until marriage and no girl-boy relationships until college or until daughters were in their late teens. Cultural actions that mothers were exposed to in their upbringing included not talking about reproductive health issues and threatening to disown girls who became pregnant before marriage. An additional noteworthy observation was that most mothers believed that their daughters 10-14 years old had no interest in boys. They believed that their daughters were very focused on their education. Because this study did not interview the daughters, it is unclear if this is indeed the case. However, this might be a naïve view that limits and delays RHE provided by mothers.

Mothers exposed to myths and taboos while growing up in Africa reported that they did not believe in them. Some mothers mentioned that they did not believe the myths and taboos because of the advanced education they had received. Of note was the fact that $50 \%$ of the sample were either nurses or worked in healthcare. It was however crucial that this study examined myths and taboos because review of literature in select African countries indicated that they (myths and taboos) influenced reproductive health communication. These mothers provided RHE to their daughters without passing on myths or taboos.

The exception was Lola, who reported that she wanted to pass on myths and taboos to her daughter because it was part of her culture-one she was forgetting, despite her desire to remember. She was also the only one who reported coming to the U.S. as a refugee, a situation in which there was a forced separation from her culture, which may potentially have influenced her desire to maintain all aspects of her culture-even negative aspects. Lola's response speaks to a phenomenon known as cultural bereavement, in which the loss of one's social structure, culture and cultural identity, can cause a grief reaction. Cultural bereavement especially occurs when an individual is abruptly uprooted from their environment of origin, and placed in a setting in which they may not be able to practice expression of culture, as is the case with refugees [22]. Although Lola expressed a desire to pass on more aspects of her culture, both positive and negative, her description of information passed to her daughter was factual, and provided to her daughter at an early age.

When queried, mothers acknowledged the necessity of providing RHE because life in the U.S. is different from life in African countries. These differences are seen as daughters are exposed to more sexual content in the U.S. than in the mothers' countries of origin. The mothers also stated that there are more media sources here in the U.S. through which to access potentially sexual material. They realize that conversations about RHE must occur at earlier ages in the U.S. and that these conversations may require more RHE content than they are comfortable providing.

These findings advance our understanding of the cultural context of African immigrant mothers living in the United States. Although we cannot generalize our results, we begin to understand the contexts in which the mothers were raised and how that contributed to subsequent reproductive health discussions with their daughters. The mothers in our study acknowledged that cultural differences required them to have conversations for which they had little preparation and that their cultural background forbade discussing. Despite this, living in the U.S. appears to contribute to earlier, more detailed RHE conversations among the mothers interviewed.

Myths and taboos are of particular concern because they influence behaviors and actions. Although women may not identify their cultural beliefs and practices as untrue or potentially dangerous to their health, we recommend that healthcare providers should collect information on any identified myths and taboos on health histories and attempt to educate where harmful practices are revealed.

Acknowledgements Financial support for the study described in this manuscript was provided by (1) the Sigma Theta Tau Eta Chapter Research Award, (2) the Beryl B. Haughton Jackson Endowed Fund for Graduate Students to Study Women's Health, and (3) The Center for Health Equity (CHE) Masters and Doctoral Student Scholarship Award.

\section{Compliance with Ethical Standards}

Conflict of interest The authors declare that they have no conflict of interest.

Ethical Approval All procedures performed in the study were in accordance with the ethical standards of the institutional and/or national research committee and with the 1964 Helsinki declaration and its later amendments or comparable ethical standards.

Informed Consent Informed consent was obtained from all individual participants included in the study.

\section{References}

1. Capps R, McCabe K, Fix M. New streams: Black African migration to the United States. Washington, DC: Migration Policy Institute; 2011.

2. Berry JW. Immigration, acculturation, and adaptation. Appl. Physiol. 1997;46(1):5-68.

3. Abraído-Lanza AF, Armbrister AN, Flórez KR, Aguirre AN. Toward a theory-driven model of acculturation in public health research. Am J Public Health. 2006;96(8):1342-6. https://doi. org/10.2105/AJPH.2005.064980.

4. Amoran O, Onadeko M, Adeniyi J. Parental influence on adolescent sexual initiation practices in Ibadan, Nigeria. Int Q Commun Health Educ. 2005;23(1):73-81.

5. Boakye PA. Dipo: a rite of passage among the Krobos of Eastern Region, Ghana. (Master of Philosophy in Indigenous Studies), University of Troms $\varnothing$, Norway, Department of Humanities, Social 
Sciences and Education, 2010. http://munin.uit.no/bitstream/handle $/ 10037 / 2673 /$ thesis.pdf? sequence $=2$.

6. Mathew R, Shugaba A, Ogala W. Parents-adolescents communication and HIV/AIDS in Jos North Local Government Area, Plateau State, Nigeria. J Med Sci. 2006;6:537-45.

7. Gueye A, Speizer IS, Corron M, Okigbo CC. Belief in family planning myths at the individual and community levels and modern contraceptive use in Urban Africa. Int Perspect Sex Reprod Health. 2015;41(4):191-9. https://doi.org/10.1363/4119115.

8. Sedgh G, Hussain R. Reasons for contraceptive nonuse among women having unmet need for contraception in developing countries. Stud Fam Plann. 2014;45(2):151-69.

9. Ankomah A, Anyanti J, Oladosu M. Myths, misinformation, and communication about family planning and contraceptive use in Nigeria. Open Access J Contracept. 2011;2(1):95-105.

10. Amanze J. Demythologizing African conceptions of human sexuality: a gateway to prevention and eradication of HIV and AIDS in Africa, 2010, from http://inerela.org/2010/07/demythologizingafrican-conceptions-of-human-sexuality-a-gateway-to-preventionand-eradication-of-hiv-and-aids-in-africa-prof-james-n-amanze/.

11. Izugbara C. Home-based sexuality education: Nigerian parents discussing sex with their children. Youth Soc. 2008;39(4):575600. https://doi.org/10.1177/0044118X07302061.

12. Biddlecom A, Awusabo-Asare K, Bankole A. Role of parents in adolescent sexual activity and contraceptive use in four African countries. Int Perspect Sex Reprod Health. 2009;35(2):72-81. https://doi.org/10.1363/ipsrh.35.072.09.

13. Mbugua N. Factors inhibiting educated mothers in Kenya from giving meaningful sex-education to their daughters.
Soc Sci Med. 2007;64(5):1079-89. https://doi.org/10.1016/j. socscimed.2006.10.008.

14. Nganda S. Sex education: do our teens need it? Paper presented at: Kenyatta University; 2008; Nairobi, Kenya. http://www.arsrc. org/downloads/uhsss/nganda.pdf. Accessed 14 Feb 2014.

15. Opara PI, Eke GK, Akani NA. Mothers perception of sexuality education for children. Niger J Med. 2010;19(2):168-72.

16. Agbemenu K, Devido J, Terry MA, Hannan M, Kitutu J, Doswell W. Exploring the experience of African immigrant mothers providing reproductive health education to their daughters aged 10 to 14 years. J Transcult Nurs. 2016. https://doi. org/10.1177/1043659616681848.

17. Agbemenu K, Schlenk EA. An integrative review of comprehensive sex education for adolescent girls in Kenya. J Nurs Scholarsh. 2011;43(1):54-63. https://doi. org/10.1111/j.1547-5069.2010.01382.x.

18. Wilson EK, Dalberth BT, Koo HP, Gard JC. Parents' perspectives on talking to preteenage children about sex. Perspect Sex Reprod Health. 2010;42(1):56-63. https://doi.org/10.1363/4205610.

19. Sandelowski M. Whatever happened to qualitative description? Res Nurs Health. 2000;23(4):334-340.

20. Sandelowski M, Barroso J, Voils CI. Using qualitative metasummary to synthesize qualitative and quantitative descriptive findings. Res Nurs Health. 2007;30(1):99-111.

21. Brown EJ, Jemmott LS. HIV prevention among people with developmental disabilities. J Psychosoc Nurs Ment Health Serv. 2002;40(11):14-21.

22. Bhugra D, Becker MA. Migration, cultural bereavement and cultural identity. World Psychiatry. 2005;4(1):18-24. 